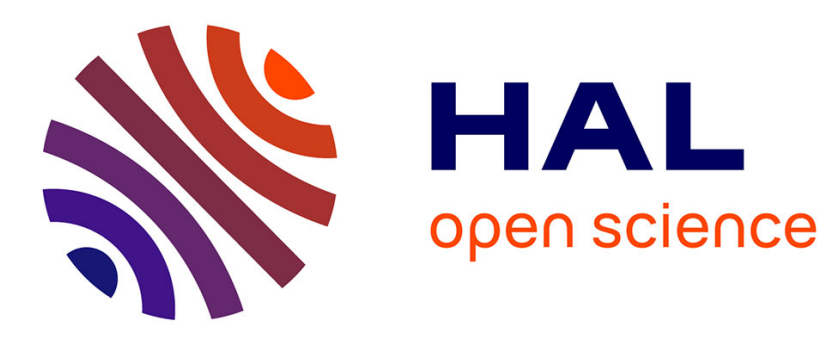

\title{
Jensen's and Wirtinger's inequalities for time-delay systems
}

Alexandre Seuret, Frédéric Gouaisbaut

\section{To cite this version:}

Alexandre Seuret, Frédéric Gouaisbaut. Jensen's and Wirtinger's inequalities for time-delay systems. 11th IFAC Workshop on Time-Delay Systems, Feb 2013, Grenoble, France. 6p. hal-00760314

\section{HAL Id: hal-00760314 \\ https://hal.science/hal-00760314}

Submitted on 3 Dec 2012

HAL is a multi-disciplinary open access archive for the deposit and dissemination of scientific research documents, whether they are published or not. The documents may come from teaching and research institutions in France or abroad, or from public or private research centers.
L'archive ouverte pluridisciplinaire HAL, est destinée au dépôt et à la diffusion de documents scientifiques de niveau recherche, publiés ou non, émanant des établissements d'enseignement et de recherche français ou étrangers, des laboratoires publics ou privés. 


\title{
Jensen's and Wirtinger's inequalities for time-delay systems
}

\author{
A. Seuret ${ }^{*, * *}$ F. Gouaisbaut ${ }^{*, * * *}$ \\ * CNRS, LAAS, 7 avenue du Colonel Roche, 31077 Toulouse, France. \\ (e-mail: aseuret,fgouaisb@laas.fr). \\ ** Univ de Toulouse, LAAS, F-31400 Toulouse, France \\ *** Univ de Toulouse, UPS, LAAS, F-31400, Toulouse, France.
}

\begin{abstract}
In the huge literature dedicated to stability of time-delay systems, the most popular approach remains the use of Lyapunov-Krasovskii functionals. This framework allows to study a large class of time-delay systems including constant or time-varying delays. Since several years, the main challenge is to propose new functionals and techniques for deriving less and less conservative stability conditions. Nevertheless, all these approaches usually adopt the same procedure which is based on the well-known Jensen's inequality which generally induces some conservatism difficult to overcome. This paper analyses firstly the conservatism induced by the Jensen's inequality and secondly proposes a wide class of new parametrized inequalities. All these are based on an extensive use of Wirtinger inequality which has been recently introduced in Liu and Fridman [2012] and Seuret and Gouaisbaut [2012b] for stability analysis.
\end{abstract}

Keywords: Time-delay systems, stability analysis, Lyapunov

\section{INTRODUCTION}

Proving stability of time-delay systems is a recurrent problem since delays are inherent in many practical problems in engineering fields and control theory (see Sipahi et al. [2011] for a recent survey). Several methods have been employed, from frequential approaches (see Sipahi et al. [2011] for a recent survey) to state space approaches using Lyapunov functionals (Gu et al. [2003]) along with robust analysis counterpart (like Small gain Theorem Niculescu and Chen [1999], Zhang et al. [2001]), IQC approach (Kao and Rantzer [2007]) or quadratic separation approach (Ariba et al. [2010]). In the case of stable linear time-delay system, a Lyapunov-Krasovskii functional can be constructed but it relies on a delay Lyapunov matrix (Kharitonov and Plischke [2006]). The parameters involved in the so-called complete Lyapunov functional are solutions of a linear differential-difference equation with boundary conditions. As it is therefore quite complicated to solve, many works have been dedicated to the construction of approximate complete Lyapunov-Krasovskii functionals. By definition, the choice of such functionals entails a certain part of conservatism and many efforts have been put on improving such results. Several ways have been therefore explored. One possible solution is to construct more and more complex Lyapunov-Krasovskii functionals by choosing extended state variables, which generally goes along with a certain numerical burden (Ariba and Gouaisbaut [2009], Kim [2011]). Often associated with these new functionals, the authors introduce extra variables called slack variables in order to relax the optimization problem, expressed in terms of LMIs. Getting numerically tractable inequalities (generally some LMIs to be optimized) have been then the core of many research since several years. In all these works, Jensen's inequality has been recognized as a powerful tool to obtain efficient results even if it is at a price of an unavoidable conservatism. Surprisingly, this has been barely studied yet except in a few papers (Briat [2011], Seuret and Gouaisbaut [2012a,b]). In Briat [2011], an upperbound of the conservatism induced by Jensen inequality is proposed by using Grüss inequality. This last result is then employed to derive equivalent new inequalities well fitted for an optimization scheme like LMIs. In Seuret and Gouaisbaut [2012a,b], the benefits of using the Wirtinger's inequalities instead of Jensen's one has been shown successfully. In particular it has been proved that the choice of a particular signal (denoted in the sequel by $z$ ) which satisfies the necessary assumptions to apply the Wirtinger inequalities, leads to a new inequality which encompasses the well-known Jensen's inequality. The resulting inequality does not only depend on the classical terms $x(t)$ or $x(t-h)$ but also on $\int_{t-h}^{t} x(s) d s$. This last signal is then directly integrated into a new suitable Lyapunov-Krasovskii functional, highlighting the features of Wirtinger inequality. Notice that this new class of inequalities has been already employed in stability of sampled-data systems by Liu and Fridman [2012]. A new Lyapunov functional is derived using directly this new inequality.

In this work, the first part is devoted to the construction of a lower bound for Jensen gap. Then, we aim at reducing the conservatism of Lyapunov-Krasovskii techniques by considering an accurate integral inequality which includes the Jensen's one as a special case : the Wirtinger's inequality. We explore the possible choices of signal $z$ which satisfies the assumptions of the Wirtinger's inequality. Based on the general formulation, our objective is to investigate in the optimal choice of the signal $z$ if possible. 
Notations: Throughout the paper $\mathbb{R}^{n}$ denotes the $n$ dimensional Euclidean space with vector norm $|\cdot|, \mathbb{R}^{n \times m}$ is the set of all $n \times m$ real matrices, and the notation $P>0$, for $P \in \mathbb{R}^{n \times n}$, means that $P$ is symmetric and positive definite. The symmetric matrix $\left[\begin{array}{ll}A & B \\ * & C\end{array}\right]$ stands for $\left[\begin{array}{cc}A & B \\ B^{T} & C\end{array}\right]$. For any differentiable $z:[a, b] \rightarrow \mathbb{R}^{n}$, we denote the norm $\|z\|_{2}^{2}=\frac{1}{b-a} \int_{a}^{b} z^{T}(u) z(u) \mathrm{d} u$.

\section{PRELIMINARIES}

Using Lyapunov-Krasovskii functionals to derive stability conditions for time-delay systems usually leads to some matrix inequalities quite difficult to optimize. Using Jensen's inequality often allows to derive useful inequality which can be directly incorporated in an optimization setup. For our purpose, let us recall the Jensen's inequality in the following lemma.

Lemma 1. For a given matrix $R>0$ and for any differentiable signal $\omega$ in $[a, b] \rightarrow \mathbb{R}^{n}$, the following inequality holds:

$$
\int_{a}^{b} \dot{\omega}^{T}(u) R \dot{\omega}(u) d u \geq \frac{(\omega(b)-\omega(a))^{T} R(\omega(b)-\omega(a))}{b-a} .
$$

Following Briat [2011], the Jensen gap $g_{R}(\omega)$ is referred as:

$$
\begin{aligned}
g_{R}(\dot{\omega}) & =\int_{a}^{b} \dot{\omega}^{T}(u) R \dot{\omega}(u) d u \\
& -\frac{(\omega(b)-\omega(a))^{T} R(\omega(b)-\omega(a))}{b-a} .
\end{aligned}
$$

In the present article, our objectives are twofold. Firstly, by employing a new class of integral inequalities called Wirtinger's inequalities, we aim at giving a lower-bound for the Jensen gap. Secondly, based on this first preliminary result, we propose some numerically tractable inequalities which can be useful for stability tests of time delays systems and sampled data systems as well. Let us recall the original Wirtinger inequality in the following lemma.

Lemma 2. Let $z$ a differentiable function and $z(a)=z(b)$ and $\int_{a}^{b} z(u) \mathrm{d} u=0$. Then for any $n \times n$ matrix $R>0$, the following inequality holds

$$
\int_{a}^{b} \dot{z}^{T}(u) R \dot{z}(u) \mathrm{d} u \geq \frac{4 \pi^{2}}{(b-a)^{2}} \int_{a}^{b} z^{T}(u) R z(u) \mathrm{d} u .
$$

There exist also other versions of the Wirtinger's inequality, which can be found in Kammler [2007], and which could be of great interests. For instance the second version of the Wirtinger's inequality proposed anther integral inequality which requires less constraints on the function $z$ to be considered. This correspond to the following lemma. Lemma 3. Let $z$ a differentiable function and $z(a)=$ $z(b)=0$. Then for any $n \times n$ matrix $R>0$, the following inequality holds

$$
\int_{a}^{b} \dot{z}^{T}(u) R \dot{z}(u) \mathrm{d} u \geq \frac{\pi^{2}}{(b-a)^{2}} \int_{a}^{b} z^{T}(u) R z(u) \mathrm{d} u .
$$

This last inequality have been recently adopted Seuret and Gouaisbaut [2012a,b] to exhibit interesting inequalities which drastically reduce the conservatism of classical Lyapunov-Krasovskii methods. In this paper, we aim at understanding how these inequalities can help to assess stability of time-delay systems. In particular, in the next section, we propose a novel inequality which is proved to be less conservative than the Jensen's one.

Remark 1. Another version of the Wirtinger inequality can be found in Liu and Fridman [2012] but will not be presented here.

The following section constructs a link between the Jensen's and the Wirtinger's inequalities. This is not straightforward, since in the Jensen's Lemma, no assumption on the considered signal are needed contrary to Wirtinger Lemma which requires strong assumptions on the signal $z$. Recall that the objectives of the present paper is to obtain new lower bounds of the integral $\int_{a}^{b} \dot{\omega}(u) R \dot{\omega}(u) \mathrm{d} u$, in order to be consistent with the Jensen's inequality. Thus a first step consists in defining appropriate function $z$ such that this integral appears naturally in the developments. Thus a necessary condition is that the function $z$ as the following form

$$
z(u)=\gamma \omega(u)-y(u)
$$

where $\gamma$ is a scalar and where $y(\cdot)$ is a vector function of appropriate dimension.

\section{HOW CONSERVATIVE JENSEN'S INEQUALITY IS?}

This section aims at showing how Jensen's inequality could be conservative by providing a lower bound for the Jensen's gap. To this end, we provide the following Corollary:

Corollary 4. For a given symmetric positive definite matrix $R>0$, any function any differentiable signal $\omega$ in $[a, b] \rightarrow \mathbb{R}^{n}$, then the following inequality holds:

$$
\begin{gathered}
\int_{a}^{b} \dot{\omega}(u) R \dot{\omega}(u) \mathrm{d} u-\frac{(\omega(b)-\omega(a))^{T} R(\omega(b)-\omega(a))}{b-a} \\
\geq \frac{4 \pi^{2}}{(b-a)^{2}} \int_{a}^{b} z^{T}(u) R z(u) \mathrm{d} u,
\end{gathered}
$$

where the function $z$ is defined as follows:

$$
\begin{aligned}
z(u)= & \omega(u)-\frac{1}{b-a} \int_{a}^{b} \omega(u) \mathrm{d} u \\
& -\left[\frac{(u-a)}{b-a}-\frac{1}{2}\right](\omega(b)-\omega(a)) .
\end{aligned}
$$

Proof : Remark that the function $z$ defined by (7) is of the form given in (5) and has been constructed in order to fulfill the assumption on the function $z$ described in Lemma 2. Then, computing the left-hand side of the inequality (3) leads to

$$
\begin{aligned}
\int_{a}^{b} \dot{z}^{T}(u) R \dot{z}(u) \mathrm{d} u & =\int_{a}^{b} \dot{\omega}^{T}(u) R \dot{\omega}(u) \mathrm{d} u \\
& -\frac{1}{(b-a)}(\omega(b)-\omega(a))^{T} R(\omega(b)-\omega(a)) .
\end{aligned}
$$

Hence, applying Lemma 2 allows to conclude.

Compared to Jensen's inequality which simply states that 
$g_{R}(\omega) \geq 0$, this previous corollary provides a new interesting lower bound of the Jensen's gap. This bound corresponds to an integral quadratic function and is positive definite. Simple calculations show that this equality can be also rewritten as follows:

$$
\begin{aligned}
\int_{a}^{b} z^{T}(u) R z(u) \mathrm{d} u & =\int_{a}^{b} \omega^{T}(u) R \omega(u) \mathrm{d} u \\
& -\frac{1}{b-a} \int_{a}^{b} \omega^{T}(u) \mathrm{d} u R \int_{a}^{b} \omega(u) \mathrm{d} u \\
& +\frac{b-a}{12}\left((\omega(b)-\omega(a))^{T} R((\omega(b)-\omega(a))\right. \\
& +\Xi
\end{aligned}
$$

where

$$
\Xi=\left(\int_{a}^{b} \omega(s) d s-\frac{2}{b-a} \int_{a}^{b} \int_{u}^{b} \omega(s) d s d u\right)^{T} R(\omega(b)-\omega(a)) .
$$

A simple inspection of this last expression shows that this may be difficult to handle especially if we are looking for numerically tractable inequalities. A naive approach to derive efficient relations could be the use of Jensen lemma on $\int_{a}^{b} z^{T}(u) R z(u) \mathrm{d} u$,

$$
\int_{a}^{b} z^{T}(u) R z(u) \mathrm{d} u \geq \frac{1}{b-a} \int_{a}^{b} z^{T}(u) \mathrm{d} u \int_{a}^{b} z(u) \mathrm{d} u,
$$

but as $\int_{a}^{b} z(u) \mathrm{d} u=0$, this last inequality does not lead to any improvements. The constraint on the signal $z$ and its integral are thus very restrictive. They lead to many difficulties when constructing other admissible signals $z$. Thus in the next section, an analysis of the possible inequalities based on the second version of the Wirtinger's inequality is provided in Lemma 3.

Remark 2. We can also draw a parallel with the result of Briat [2011], where an upperbound is given using Grüss inequality. Indeed, using Corollary 2.1 from Briat [2011], one exhibits the following inequality:

$$
g_{R}(\dot{\omega}) \leq \frac{(b-a)^{3}}{4}\left(\sup _{[a, b]}|\ddot{\omega}|\right)^{T} R\left(\sup _{[a, b]}|\ddot{\omega}|\right) .
$$

As a matter of fact, we get the following result:

Corollary 5. The Jensen gap is such that:

$$
\begin{aligned}
& \frac{4 \pi^{2}}{(b-a)^{2}} \int_{a}^{b} z^{T}(u) R z(u) \mathrm{d} u \leq g_{R}(\dot{\omega}) \\
& \leq \frac{(b-a)^{3}}{4}\left(\sup _{[a, b]}|\ddot{\omega}|\right)^{T} R\left(\sup _{[a, b]}|\ddot{\omega}|\right),
\end{aligned}
$$

where the function $z$ is defined by $(7)$.

\section{APPLICATION OF THE SECOND WIRTINGER'S INEQUALITY}

This section focusses on exploiting the second version of the Wirtinger's inequality given in Lemma 3. As it is mentioned above, the use of the second version of the Wirtinger's inequality leads to a additional flexibility in the definition of the function $z$ employed in this lemma. Thus, in the following, we will propose a general method to construct such class of function $z$ of the form (5).

Consider a scalar function $f$ defined over $[a, b]$. Then define the associated function (5) given by

$$
\begin{aligned}
z(u)= & (f(b)-f(u)) \omega(a) \\
+ & (f(u)-f(a)) \omega(b) \\
& -(f(b)-f(a)) \omega(u),
\end{aligned}
$$

where $u \in[a, b]$. By construction, the function $z(u)$ satisfies the conditions of Lemma 3, i.e. $z(a)=z(b)=0$.

In the sequel, the following issues will be addressed.

- For a such class of functions $f$, is it possible to derive an inequality related to the Jensen Lemma?

- Is it possible to find an optimal function $\bar{f}$ which ensures that the resulting inequality is less conservative than the ones obtained by any other functions $f$ ?

The answers to these questions are given below.

\subsection{A second inequality}

The following lemma is proposed.

Lemma 6. For given matrix $R>0$ and differentiable signal $\omega$ in $[a, b] \rightarrow \mathbb{R}^{n}$ and a twice differentiable function $[a, b] \rightarrow \mathbb{R}$ such that $f(b) \neq f(a)$, the following inequality holds:

$$
\begin{aligned}
\int_{a}^{b} \dot{\omega}(u) R \dot{\omega}(u) \mathrm{d} u \geq & \alpha_{1}(f) \frac{\eta^{T}(a, b) R \eta^{T}(a, b)}{b-a} \\
& +\pi^{2} \frac{\nu^{T}(a, b) R \nu^{T}(a, b)}{b-a}
\end{aligned}
$$

where

$$
\begin{aligned}
\eta(a, b) & =\omega(b)-\omega(a) \\
\nu(a, b) & =\left(1+\alpha_{2}(f)\right) \omega(b)-\alpha_{2}(f) \omega(a)-\int_{a}^{b} \frac{\omega(u)}{b-a} \mathrm{~d} u,
\end{aligned}
$$

where

$$
\begin{aligned}
\alpha_{1}(f)= & \frac{b-a}{f(b)-f(a)}\left(\|\dot{f}\|_{2}-\frac{(b-a)^{2}}{\pi^{2}}\|\ddot{f}\|_{2}\right), \\
\alpha_{2}(f)= & \frac{1}{f(b)-f(a)}\left(\frac{\left(\int_{a}^{b} f(u) \mathrm{d} u\right)}{b-a}\right. \\
& \left.+\frac{(b-a)}{\pi}(\dot{f}(b)-\dot{f}(a))-f(b)\right) .
\end{aligned}
$$

Proof: Let $f$ be a continuous and twice differentiable scalar function $f$ defined over $[a, b]$ such that $f(a) \neq f(b)$. As suggested previously consider the signal $z$ given by

$$
\begin{aligned}
z(u)= & (f(b)-f(u)) \omega(a) \\
& +(f(u)-f(a)) \omega(b) \\
& -(f(b)-f(a)) \omega(u),
\end{aligned}
$$

where $u \in[a, b]$.

Since $z(a)=z(b)=0$, Lemma 3 can be applied to this signal. This leads to:

$$
\int_{a}^{b} \dot{z}^{T}(u) R \dot{z}(u) \mathrm{d} u \geq \frac{4 \pi^{2}}{(b-a)^{2}} \int_{a}^{b} z^{T}(u) R z(u) \mathrm{d} u .
$$

The right-hand-side of Lemma 3 is rewritten as:

$$
\begin{gathered}
\int_{a}^{b} \dot{z}^{T}(u) R \dot{z}(u) \mathrm{d} u=(f(b)-f(a))^{2} \int_{a}^{b} \dot{\omega}^{T}(u) R \dot{\omega}(u) \mathrm{d} u \\
\quad+\left(\int_{a}^{b} \dot{f}^{2}(u) \mathrm{d} u\right)(\omega(b)-\omega(a))^{T} R(\omega(b)-\omega(a)) \\
\quad-2(f(b)-f(a))(\omega(b)-\omega(a))^{T} R \int_{a}^{b} \dot{f}(u) \dot{\omega}(u) \mathrm{d} u
\end{gathered}
$$


An integration by parts of the last term leads to

$\int_{a}^{b} \dot{f}(u) \dot{\omega}(u) \mathrm{d} u=\dot{f}(b) \omega(b)-\dot{f}(a) \omega(a)-\int_{a}^{b} \ddot{f}(u) \omega(u) \mathrm{d} u$.

Combining the previous expression with (9) yields

$$
\begin{aligned}
\int_{a}^{b} \dot{z}^{T}(u) R \dot{z}(u) \mathrm{d} u= & (f(b)-f(a))^{2} \int_{a}^{b} \dot{\omega}^{T}(u) R \dot{\omega}(u) \mathrm{d} u \\
& +\Psi
\end{aligned}
$$

where

$$
\begin{aligned}
\Psi= & \left(\int_{a}^{b} \dot{f}^{2}(u) \mathrm{d} u\right)(\omega(b)-\omega(a))^{T} R(\omega(b)-\omega(a)) \\
& +2(f(b)-f(a))(\omega(b)-\omega(a))^{T} R \times \\
& \left(\int_{a}^{b} \ddot{f}(u) \omega(u) \mathrm{d} u-[\dot{f}(b) \omega(b)-\dot{f}(a) \omega(a)]\right) .
\end{aligned}
$$

Introduce the vector

$$
\xi(u)=\frac{\pi}{(b-a)} z(u)+\frac{(b-a)}{\pi} \ddot{f}(u)(\omega(b)-\omega(a)) .
$$

Following this definition, we have

$$
\begin{aligned}
& \int_{a}^{b} \xi^{T}(u) R \xi(u) \mathrm{d} u=\frac{\pi^{2}}{(b-a)^{2}} \int_{a}^{b} z^{T}(u) R z(u) \mathrm{d} u \\
& \quad+\frac{(b-a)^{2}\left(\int_{a}^{b} \ddot{f}^{2}(u) \mathrm{d} u\right)}{\pi^{2}}(\omega(b)-\omega(a))^{T} R(\omega(b)-\omega(a)) \\
& \quad+2(\omega(b)-\omega(a))^{T} R \int_{a}^{b} \ddot{f}(u) z(u) \mathrm{d} u
\end{aligned}
$$

The following developments focus on the last term of (11). Performing an integration by parts yields to

$$
\begin{aligned}
\int_{a}^{b} \ddot{f}(u) z(u) \mathrm{d} u= & (f(b)-f(a))[\dot{f}(b) \omega(b)-\dot{f}(a) \omega(a)] \\
& -\left(\int_{a}^{b} \dot{f}^{2}(u) \mathrm{d} u\right)(\omega(b)-\omega(a)) \\
& -(f(b)-f(a)) \int_{a}^{b} \ddot{f}(u) \omega(u) \mathrm{d} u .
\end{aligned}
$$

Combining the previous expression with (11) yields to

$$
\begin{aligned}
\int_{a}^{b} \xi^{T}(u) R \xi(u) \mathrm{d} u=\frac{\pi^{2}}{(b-a)^{2}} \int_{a}^{b} z^{T}(u) R z(u) \mathrm{d} u-\Psi \\
-\frac{\alpha_{1}(f)(f(b)-f(a))^{2}}{(b-a)}(\omega(b)-\omega(a))^{T} R(\omega(b)-\omega(a)) .
\end{aligned}
$$

By combining (9) and (12) in order to eliminate $\Psi$, we obtain

$$
\begin{aligned}
& (f(b)-f(a))^{2} \int_{a}^{b} \dot{\omega}^{T}(u) R \dot{\omega}(u) \mathrm{d} u= \\
& \quad \frac{\alpha_{1}(f)}{(b-a)}(f(b)-f(a))^{2}(\omega(b)-\omega(a))^{T} R(\omega(b)-\omega(a)) \\
& \quad+\int_{a}^{b} \xi^{T}(u) R \xi(u) \mathrm{d} u \\
& \quad+\int_{a}^{b} \dot{z}^{T}(u) R \dot{z}(u) \mathrm{d} u-\frac{\pi^{2}}{(b-a)^{2}} \int_{a}^{b} z^{T}(u) R z(u) \mathrm{d} u .
\end{aligned}
$$

Then Lemma 3 and Jensen's inequality ensure that the following inequality holds

$$
\begin{aligned}
\int_{a}^{b} \dot{\omega}^{T}(u) R \dot{\omega}(u) \mathrm{d} u \geq \\
\quad \frac{1}{(b-a)}\left[\alpha_{1}(f)(\omega(b)-\omega(a))^{T} R(\omega(b)-\omega(a))\right. \\
\left.\quad+\frac{1}{(f(b)-f(a))^{2}} \int_{a}^{b} \xi^{T}(u) d u R \int_{a}^{b} \xi(u) \mathrm{d} u\right]
\end{aligned}
$$

The last step of the proof consists in the computation of the integral $\int_{a}^{b} \xi^{T}(u) \mathrm{d} u$, which is obtained by performing an integration by parts as follows

$$
\begin{aligned}
& \int_{a}^{b} \xi^{T}(u) \mathrm{d} u= \\
& \quad \frac{\pi}{b-a} \int_{a}^{b}[(f(u)-f(a)) \omega(b)+(f(b)-f(u)) \omega(a)] \mathrm{d} u \\
& \quad-\frac{\pi}{b-a}(f(b)-f(a)) \int_{a}^{b} \omega(u) \mathrm{d} u \\
& \quad+\frac{(b-a)}{\pi} \int_{a}^{b} \ddot{f}(u) \mathrm{d} u(\omega(b)-\omega(a)), \\
& =\pi\left(\frac{\int_{a}^{b} f(u) \mathrm{d} u}{b-a}+\frac{(b-a)}{\pi^{2}}(\dot{f}(b)-\dot{f}(a))\right)(\omega(b)-\omega(a)) \\
& -\pi \frac{(f(b)-f(a))}{b-a} \int_{a}^{b} \omega(u) \mathrm{d} u-\pi(f(a) \omega(b)-f(b) \omega(a)) .
\end{aligned}
$$

Finally we get that

$$
\int_{a}^{b} \xi^{T}(u) \mathrm{d} u=\pi(f(b)-f(a)) \nu(a, b)
$$

where $\nu(a, b)$ is defined in the statements of the Lemma. This allows to get the inequality proposed in Lemma $6 . \diamond$

The previous lemma presents a wide class of inequalities. Depending on the choice of the function $f$, the coefficients $\alpha_{1}$ and $\alpha_{2}$ may have different values. In the following section, a maximization of the coefficients is proposed.

\subsection{How to encompass Jensen's inequality?}

It is relevant to see if one may find the function which reduces the conservatism of the inequality. As the first step, the proposition is derived.

Proposition 7 . For any $\beta \in \mathbb{R}^{+}, \gamma \in \mathbb{R}$ and any function $f$ satisfying the conditions of Lemma 6 , then $\alpha_{1}(\beta f+$ $\gamma)=\alpha_{1}(f)$ and $\alpha_{2}(\beta f+\gamma)=\alpha_{2}(f)$.

Proof: The proof is performed by simple computations. $\diamond$

Finding the function $f$ which maximizes the right-hand side of (8) is quite ambitious and difficult. This is the reason why we propose to investigate only in the maximization of the coefficient $\alpha_{1}(f)$. This is achieved in the following proposition.

Proposition 8. For any twice differentiable function $f$, $\alpha_{1}(f) \leq 1$ and the equality holds when $f$ is an affine function.

Proof : Consider a scalar function $f$ which satisfies the conditions of Lemma 6 . Using the Fourier series of the function $\dot{f}$, the Parseval identity ensures that 


$$
\begin{aligned}
& \frac{1}{b-a} \int_{a}^{b} \dot{f}^{2}(u) \mathrm{d} u=a_{0}^{2}+\frac{1}{2} \sum_{i \in \mathbb{N} \backslash\{0\}}\left(a_{i}^{2}+b_{i}^{2}\right), \\
& \frac{1}{b-a} \int_{a}^{b} \ddot{f}^{2}(u) \mathrm{d} u=\frac{2 \pi^{2}}{(b-a)^{2}} \sum_{i \in \mathbb{N} \backslash\{0\}} n^{2}\left(a_{i}^{2}+b_{i}^{2}\right),
\end{aligned}
$$

where

$$
\begin{aligned}
& a_{0}=\frac{1}{b-a} \int_{a}^{b} \dot{f}(u) d u=\frac{f(b)-f(a)}{b-a}, \\
& a_{i}=\frac{2}{b-a} \int_{a}^{b} \dot{f}(u) c_{i}(u) d u \\
& b_{i}=\frac{2}{b-a} \int_{a}^{b} \dot{f}(u) s_{i}(u) d u \\
& s_{i}(u)=\sin \left(\frac{2 i \pi}{b-a}(u-a)\right) \\
& c_{i}(u)=\cos \left(\frac{2 i \pi}{b-a}(u-a)\right) .
\end{aligned}
$$

Hence the coefficient $\alpha_{1}(f)$ can be rewritten as

$$
\alpha_{1}(f)=\left[1+\sum_{i \in \mathbb{N} \backslash\{0\}}\left(1-4 i^{2}\right) \frac{\left(a_{i}^{2}+b_{i}^{2}\right)}{2 a_{0}^{2}}\right] .
$$

Since $1-4 i^{2}$ is always negative for any non negative integer $i$, the coefficient $\alpha_{1}(f)$ is maximized when the Fourier coefficients $a_{n}$ and $b_{n}$ of $\dot{f}$ are zero for all integer $n>0$. This implies that the function $f$ is an affine function. Moreover, according to Proposition 7, any affine scalar functions yields the same result, i.e. $\alpha_{1}(f(u)=u)$. This concludes the proof.

According to the previous proposition, the following corollary is provided.

Corollary 9. For a given symmetric positive definite matrix $R>0$, any function any differentiable signal $\omega$ in $[a, b] \rightarrow \mathbb{R}^{n}$ and any twice differentiable function $[a, b] \rightarrow$ $\mathbb{R}$, then the following inequality holds:

$$
\begin{aligned}
\int_{a}^{b} \dot{\omega}(u) R \dot{\omega}(u) \mathrm{d} u \geq & \frac{\eta^{T}(a, b) R \eta(a, b)}{b-a} \\
& +\frac{\nu_{0}^{T}(a, b) R \nu_{0}(a, b)}{b-a},
\end{aligned}
$$

where $\eta(a, b)$ is defined in Lemma 6 and

$$
\nu_{0}(a, b)=\frac{\omega(b)+\omega(a)}{2}-\frac{1}{b-a} \int_{a}^{b} \omega(u) \mathrm{d} u .
$$

Proof : Simple computations yield $\alpha_{1}(f(u)=u)=1$ and $\alpha_{2}(f(u)=u)=-\frac{1}{2}$ which conclude the proof.

Remark 3. The previous lemma has already been presented in the Seuret and Gouaisbaut [2012b]. However the optimality of the choice of the function $f$ has not been discussed there. Thus the previous contributions aim at showing this first choice was the optimal one regarding the particular definition of the function $z$. Of course, the present paper does not provide an optimal choice of $f$ for both coefficients $\alpha_{1}$ and $\alpha_{2}$ but just gives a clue of which function would be suitable to consider.

Remark 4. As shown in Corollary 4, the previous corollary provides a lower bound on the Jensen's gap. Indeed the previous inequality can be rewritten as follows

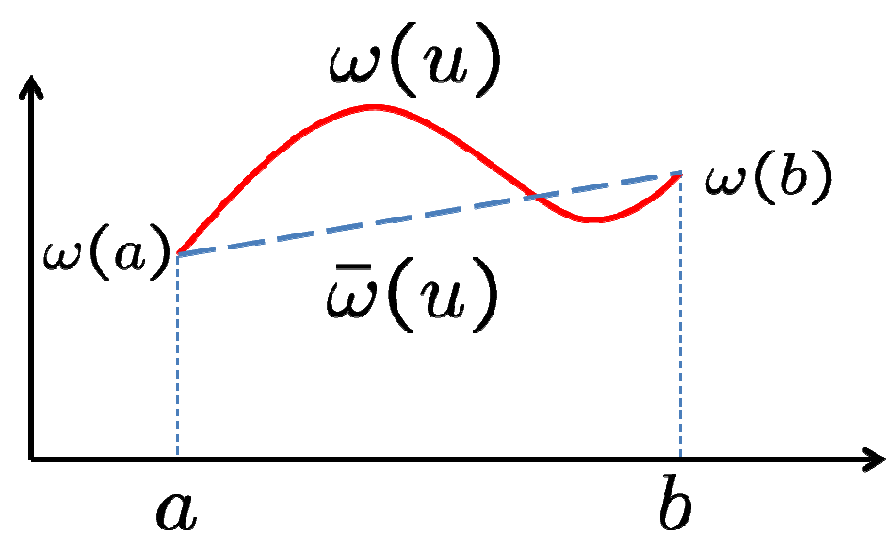

Fig. 1. Graph representing a signal $\omega$ and its interpolating polynomial $\bar{\omega}$

$$
g_{r}(\dot{\omega}) \geq \frac{\nu_{0}^{T}(a, b) R \nu_{0}(a, b)}{b-a}
$$

\subsection{Some additional remarks}

As it was mentioned in the previous section, Corollary 9 provides an more accurate inequality than the Jensen inequality. Despite of this main characteristic, several additional comments on the signal $z$ can be added. When $f$ is the identity function, the function $z$ becomes

$$
z(u)=(b-a)\left(\frac{u-a}{b-a} \omega(b)+\frac{b-u}{b-a} \omega(a)-\omega(u)\right) .
$$

Note that this signal corresponds to the error of an arbitrary signal $\omega$ and its Lagrange interpolating polynomial of degree 1 considered over the interval $[a, b]$ and given by

$$
\bar{\omega}(u)=\frac{u-a}{b-a} \omega(b)+\frac{b-u}{b-a} \omega(a) .
$$

This is represented in Figure 1. Thus it would be interesting, in future works, to investigate other interpolating polynomials with, for instance, a higher degree or considering additional information of the signal $\omega$ over the interval $[a, b]$ as, for instance, $\omega((a+b) / 2)$. This last consideration refers some how to a discretization method.

Following this idea, an other important remark concerns the resulting signal $\nu(a, b)$. Recall that in Corollary 9, this signal becomes

$$
\nu(a, b)=\frac{1}{b-a}\left[(b-a) \frac{\omega(b)-\omega(a)}{2}-\int_{a}^{b} \omega(u) \mathrm{d} u\right] .
$$

This term corresponds to the approximation error of the integral of an arbitrary signal $\omega$ and the area enclosed in the trapeze defined by the $\omega(a)$ and $\omega(b)$ and the abscissa axis as shown in Figure 2. This last remark is completely in accordance with the trapezoidal rule using Lagrange polynomial of order 1 . As a matter of fact, there exists other relevant methods to approximate the integral of a function over an interval of the form $[a, b]$ and it should be interesting to investigate on these other methods which could deliver improved results. 


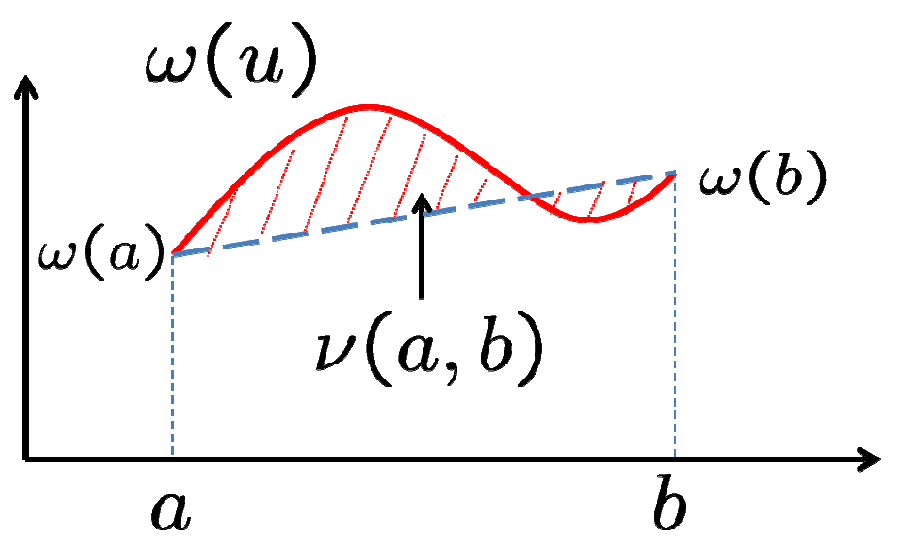

Fig. 2. Graphical representation of $\nu(a, b)$ for an arbitrary signal $\omega$.

\section{CONCLUSIONS}

This paper investigates the use of a new inequality called Wirtinger inequality for stability purpose. Firstly, this inequality is linked to the Jensen inequality and provides some explanations on the conservatism induced by the use of Jensen Lemma. Secondly, we use this new inequality to establish some new numerically tractable inequalities which outperform the well known Jensen inequality often used in the context of time delay systems / sampled data stability.

\section{REFERENCES}

Y. Ariba and F. Gouaisbaut. An augmented model for robust stability analysis of time-varying delay systems. Int. J. Control, 82:1616-1626, September 2009.

Y. Ariba, F. Gouaisbaut, and K.H. Johansson. Stability interval for time-varying delay systems. In Decision and Control $(C D C), 2010$ 49th IEEE Conference on, pages $1017-1022$, dec. 2010.

C. Briat. Convergence and equivalence results for the Jensen's inequality - application to time-delay and sampled-data systems. IEEE Transactions on Automatic Control, 56(7):1660-1665, 2011.

K. Gu, V.-L. Kharitonov, and J. Chen. Stability of timedelay systems. Birkhauser, 2003.

W.D. Kammler. A first Course in Fourier Analysis. Cambridge University Press, Cambridge, 2007.

C.Y. Kao and A. Rantzer. Stability analysis of systems with uncertain time-varying delays. Automatica, 43(6): $959-970,2007$.

V.L. Kharitonov and E. Plischke. Lyapunov matrices for time-delay systems. Systems Eamp; Control Letters, 55 (9):697 - 706, 2006. ISSN 0167-6911.

J.H. Kim. Note on stability of linear systems with timevarying delay. Automatica, 47(9):2118-2121, 2011.

K. Liu and E. Fridman. Wirtinger's inequality and lyapunov-based sampled-data stabilization. Automatica, 48(1):102108, 2012.

S. I. Niculescu and J Chen. Frequency sweeping tests for asymptotic stability: a model transformation for multiple delays. In IEEE Conference on Decision and Control, pages 4678-4683, December 1999.

A. Seuret and F. Gouaisbaut. Reducing the gap of the jensen's inequality by using the wirtinger's inequality. submitted to Automatica, 2012a.
A. Seuret and F. Gouaisbaut. On the use of the wirtinger's inequalities for time-delay systems. In Proc. of the $10^{\text {th }}$ IFAC Workshop on Time Delay Systems (IFAC TDS'12), Boston, MA, USA, 2012b.

R. Sipahi, S. Niculescu, C.T. Abdallah, W. Michiels, and Keqin Gu. Stability and stabilization of systems with time delay. Control Systems, IEEE, 31(1):38 -65, feb. 2011.

J. Zhang, C. R. Knopse, and P. Tsiotras. Stability of time-delay systems: Equivalence between Lyapunov and scaled small-gain conditions. IEEE Trans. on Automat. Control, 46(3):482-486, March 2001. 